\title{
Factores pronósticos de sobrevida alejada en cáncer gástrico. Introducción del nuevo índice $\mathrm{N}+/ \mathrm{T}$
}

\author{
Manuel Figueroa-Giralt ${ }^{1}$
}

Trabajo de Ingreso a la Sociedad de Cirujanos de Chile

\section{Prognostic factors of long term survival in gastric cancer. Introduction of the new $\mathrm{N}+/ \mathrm{T}$ index}

Background: The identification of survival prognostic factors for gastric cancer, allows us to create clinical guidelines. Chile has a deficit in the analysis of long-term survival prognostic factors. Aim: To assess different prognostic factors of long-term survival in gastric cancer. Determine the survival rate at 5 and 10-years post gastrectomy, and the value of a new prognostic factor of long-term survival called N+/T. Material and Method: Prospective study of the oncological database of the Clinical Hospital of the University of Chile between May 2004 and May 2012. Results: A total of 284 patients were included, $65.4 \%$ were men and the mean age was 64.5 years. Seventy-five percent were advanced gastric cancer, $72.5 \%$ of the patients required a total gastrectomy. The lymphadenectomy practiced was D2 in $85.2 \%$, and average lymph node harvest was 30 lymph nodes. The postoperative morbidity and mortality was $17.2 \%$ and $1.7 \%$ respectively. The average global survival was 69.9 months, the 5-year survival was $56.9 \%$ and the 10 -year survival was $53.4 \%$. The $\mathrm{N}+/ \mathrm{T}$ index presented a statistically significant difference in the global survival of all the subgroups $(p<0.0001)$. The multivariate analysis showed that the significant variables were: $\mathrm{N}+/ \mathrm{T}$ index $(\mathrm{p}=0.0001$, OR: 1.1 [1.05-1.12]), LNR ( $\mathrm{p}=0.0001$, OR: 5.8 [1.04-15.6]), age $(\mathrm{p}=0.008$, OR: 1.03 [1.00-1.06]), lymphovascular permeation $(\mathrm{p}=0.0001$, OR: 2.19 [1.49-3.23]), T classification ( $\mathrm{p}=0.03$, OR: 3.4 [1.10-8.93]), N classification( $\mathrm{p}=0.001$, OR: 1.06 [1.02-1.10]), and TNM stage $(p=0.004$, OR: 1.03 [1.01-1.06]). The areas under the ROC curves of the N+/T, LNR and T classification, were $0.789,0.786$ and 0.790 respectively $(p=0.96)$. Conclusion: The independent prognostic factors of long-term survival were $\mathrm{N}+/ \mathrm{T}$ index, LNR, age, lymphovascular permeation, $\mathrm{T}$ classification, $\mathrm{N}$ classification and TNM stage. Concomitantly, a new prognostic factor has been created to assess survival in gastric cancer, the $\mathrm{N}+/ \mathrm{T}$ index.

Key words: gastric cancer; survival; prognosis; index.

\section{Resumen}

Introducción: La identificación de factores pronósticos del cáncer gástrico, ha permitido predecir la evolución de los pacientes y así tomar decisiones terapéuticas. En Chile existe un déficit en el análisis de factores pronósticos de sobrevida alejada. Objetivos: Los objetivos de este estudio fueron: evaluar distintos factores pronósticos de sobrevida alejada en cáncer gástrico, determinar la tasa de sobrevida global mayor a 5 y 10 años posoperatoria tanto en cánceres incipientes como avanzados y evaluar el valor de un nuevo factor pronóstico de sobrevida alejada denominado N+/T. Material y Método: Estudio prospectivo de la base de datos oncológica del Hospital Clínico de la Universidad de Chile entre mayo de 2004 y mayo de 2012. Resultados: Se incluyeron un total de 284 pacientes, $65,4 \%$ fueron hombres, la edad media fue 64,5 años. $75 \%$ de la muestra fueron cánceres avanzados, 72,5\% de los pacientes requirieron una gastrectomía total. La linfadenectomía practicada fue D2 en un 85,2\%. La cosecha linfononodal global media fueron 30 linfonodos. La morbilidad y mortalidad quirúrgica posoperatoria fue de 17,2\% y 1,7\% respectivamente. La sobrevida global media fue de 69,9 meses, la sobrevida a 5 años fue de $56,9 \%$ y la sobrevida a 10 años fue de 53,4\%. Al analizar el índice $\mathrm{N}+/ \mathrm{T}$, se identifica una diferencia estadísticamente significativa en la sobrevida global alejada de todos los subgrupos $(\mathrm{p}<0,0001)$. El análisis multivariado de los factores
'Departamento de Cirugía Hospital Clínico de la Universidad de Chile. Santiago, Chile.

Recibido el 6 de noviembre de 2017 y aceptado para publicación el 4 de diciembre de 2017.

Correspondencia a: Dr. Manuel Figueroa-Giralt manuelfigueroa.gi@gmail. com 
pronósticos objetiva que las variables significativas son: índice $\mathrm{N}+/ \mathrm{T}$ ( $\mathrm{p}=0,0001, \mathrm{OR}: 1,1$ [1,05-1,12]), LNR ( $\mathrm{p}=0,0001$, OR: $5,8[1,04-15,6])$, edad ( $\mathrm{p}=0,008$, OR: 1,03 [1,00-1,06]), permeación linfovascular ( $\mathrm{p}=0,0001$, OR: 2,19 [1,49-3.23]), clasificación T ( $\mathrm{p}=0,03$, OR: 3,4 [1,10-8,93]), clasificación $\mathrm{N}$ $(p=0,001$, OR: $1,06[1,02-1,10])$ y estadio TNM ( $p=0,004$, OR: 1,03 [1,01-1,06]). Las curvas ROC del índice $\mathrm{N}+/ \mathrm{T}$, LNR y clasificación T poseen áreas bajo la curva de $0,789,0,786$ y 0,790 respectivamente, sin diferencia estadística significativa $(\mathrm{p}=0,96)$. Conclusión: Los factores pronósticos independientes de sobrevida mayor a 5 años son: índice $\mathrm{N}+/ \mathrm{T}$, LNR, edad, permeación linfovascular, clasificación T, clasificación $\mathrm{N}$ y estadio TNM. Concomitantemente se ha logrado aportar un nuevo cuociente pronóstico en la evaluación de pacientes con adenocarcinoma gástrico resecados con intención curativa, el índice N+/T. Palabras clave: cáncer gástrico; sobrevida; pronóstico; índice.

\section{Introducción}

El cáncer gástrico en Chile ha experimentado un significativo cambio en cuanto a tratamiento y pronóstico en los últimos 40 años. Es así como se ha presenciado un aumento de pacientes con cánceres indiferenciados (en especial el tipo anillo en sello), tumores de tercio superior del estómago, una mayor proporción de gastrectomías totales, junto con una significativa disminución de mortalidad quirúrgica y mejora en sobrevida global a 5 años (SVg5) $)^{1-4}$.

La identificación de algunos factores pronósticos ha permitido predecir qué pacientes tendrán una peor evolución y así contribuir en la toma de decisiones terapéuticas ${ }^{5}$. Sin embargo, en nuestra realidad nacional existe un déficit de estudios que analicen factores pronósticos de sobrevida alejada. ${ }^{6}$.El objetivo principal de este estudio fue evaluar distintos factores pronósticos de sobrevida alejada en cáncer gástrico. Los objetivos secundarios son: a) describir las características demográficas de la población estudiada, b) analizar la evolución posoperatoria de los pacientes, c) determinar la tasa de sobrevida global mayor a 5 y 10 años posoperatoria y d) evaluar el valor de un nuevo factor pronóstico de sobrevida alejada denominado $\mathrm{N}+/ \mathrm{T}$.

\section{Material y Método}

Este trabajo es un análisis prospectivo de la base de datos oncológica del Hospital Clínico de la Universidad de Chile. El período de análisis fue entre mayo de 2004 y mayo de 2012, con el objeto de tener un seguimiento mayor a 5 años en todos los pacientes vivos de ese período de tiempo.

\section{Pacientes estudiados}

En el presente estudio se incluyeron todos los pacientes con adenocarcinoma gástrico y de unión gastroesofágica Siewert II y III en población adulta, tratados en forma quirúrgica con intención curativa. Se incluyeron gastrectomías subtotales, totales y ampliadas. Estas últimas se definieron como aquellas gastrectomías que incluyeron resección adicional de otro órgano para alcanzar el R0, como esófago intratorácico, colon, páncreas y riñón.

\section{Técnica quirúrgica}

Las cirugías realizadas fueron gastrectomías subtotales en caso de lesiones distales sin células en anillo de sello, o gastrectomías totales en caso de tener un carcinoma de tipo intestinal del tercio medio o proximal o un carcinoma difuso de cualquier localización. Se obtuvo un margen distal a 2 $\mathrm{cm}$ del píloro y el margen proximal a $4 \mathrm{~cm}$ en caso de lesiones incipientes y $6 \mathrm{~cm}$ en caso de lesiones avanzadas, lo que corresponde a una cirugía R0. En todos los pacientes se realizó biopsia contemporánea intraoperatoria para confirmar bordes de sección libres de compromiso tumoral. La linfadenectomía fue D2, excepto en casos de lesiones incipientes donde se realizó D1 alfa o beta. La omentectomía mayor y menor fue de regla en casos de lesiones avanzadas, mientras que en casos de lesiones incipientes se realizaron omentectomías parciales a $6 \mathrm{~cm}$ de arcada gastromental. En las lesiones de cara posterior con compromiso de serosa se realizó bursectomía. La esplenectomía se llevó a cabo en caso de compromiso linfonodal del grupo $10 \mathrm{o}$ ante invasión tumoral esplénica. En caso de lesiones con compromiso de órganos adyacentes por contigüidad (no metastásico) se realizó una gastrectomía ampliada para alcanzar R0.

\section{Definiciones}

a) La clasificación TNM y el estadio final, se estandarizó empleando la $7^{\mathrm{a}}$ ed. de la AJCC para todos los pacientes. 
T Tx: no se puede evaluar el tumor primario.

T0: sin evidencia de tumor primario en el estómago.

Tis: cáncer in situ.

T1a: tumor se extiende hasta la mucosa.

T1b: tumor se extiende hasta la submucosa.

T2: tumor se extiende hasta la muscular propia.

T3: tumor se extiende hasta la subserosa.

T4a: tumor compromete la serosa gástrica.

T4b: tumor compromete órganos que rodean el estómago.

N Nx: no se puede evaluar el compromiso linfonodal.

N0: sin compromiso linfonodal.

$\mathrm{N} 1$ : compromiso oncológico histológico de 1-2 linfonodos.

N2: compromiso oncológico histológico de 3-6 linfonodos.

N3a: compromiso oncológico histológico de 7-15 linfonodos.

N3b: compromiso oncológico histológico de 16 o más linfonodos.

M Mx: metástasis no evaluables.

M0: sin metástasis viscerales ni peritoneales.

M1: presencia de metástasis viscerales y/o peritoneales.

\section{Estadios}

Los estadios se clasificaron según la combinación de los distintos factores del TNM de la siguiente manera, siguiendo la $7^{\mathrm{a}}$ ed. de la AJCC.

0 : TisNOM0.

IA: T1N0M0.

IB: T2N0M0, T1N1M0.

IIA: T3N0M0, T2N1M0, T1N2M0.

IIB: T4aN0M0, T3N1M0, T2N2M0, T1N3M0.

IIIA: T4aN1M0, T3N2M0, T2N3M0.

IIIB: T4bN0M0, T4bN1M0, T4aN2M0, T3N3M0.

IIIC: T4bN2M0, T4bN3M0, T4aN3M0.

IV: Cualquier T, Cualquier N, M1.

b) El índice linfonodal (LNR) definido como el cuociente entre el número de linfonodos comprometido por metástasis histológica y el número total de linfonodos cosechados (expresado en porcentaje), se subdividió en 0: $0 \%$ de linfonodos comprometidos, 1: de $1 \%$ a $9 \%$ de linfonodos comprometidos, 2: de 10 a $25 \%$ de linfonodos comprometidos y 3 : más de $25 \%$ de linfonodos comprometidos ${ }^{7}$.

c) El índice $\mathrm{N}+/ \mathrm{T}$ calcula el cuociente entre el número de linfonodos positivos para metástasis de adenocarcinoma y la clasificación $\mathrm{T}$ del paciente.
Para fines matemáticos las subdivisiones de T1a y $\mathrm{T} 1 \mathrm{~b}$ se asumieron indistintamente como 1 en el denominador (ejemplos: 1/T1a $=1 / 1=1,6$ / $\mathrm{T} 3=6 / 3=2,24 / \mathrm{T} 4=24 / 4=6)$. Los resultados de la razón se dividieron en subgrupos $\mathrm{N}+/ \mathrm{T}$ A $(0), \mathrm{N}+/ \mathrm{T} \mathrm{B}(0,1-3)$ y N+/T C (>3).

d) La mortalidad quirúrgica se definió desde el momento de la cirugía hasta 30 días posoperatorios.

e) La sobrevida global se definió desde el alta hospitalaria, eliminando la mortalidad quirúrgica.

f) La sobrevida alejada se definió como aquella mayor a 5 años posoperatorios.

g) El tiempo cero para determinar asociación pronostica fue la gastrectomía.

h) Las comorbilidades fueron definidas a partir de las guías ministeriales chilenas MINSAL $^{8-11}$.

\section{Cálculos estadísticos}

Los factores pronósticos evaluados fueron demográficos (sexo, edad, clasificación ASA, comorbilidades), clínicos (síntomas, exámenes de laboratorio), quirúrgicos (tipo de gastrectomía, morbilidad quirúrgica, morbilidad médica), anatomopatológicos (presencia de células en anillo de sello, permeación linfovascular, compromiso perineural, clasificación TNM y estadios de dicha clasificación), e índices pronósticos (índice linfonodal e índice $\mathrm{N}+/ \mathrm{T}$ ).

La distribución de las variables se determinó por test de Shapirowilk. De acuerdo a este, las variables continuas con distribución paramétrica (ordinal) se expresan en promedio y desviación estándar (DS), mientras que en las variables continuas con distribución no paramétricas (nominal) se empleó mediana y rango intercuartil $\left(\mathrm{IC}_{25 \%-75 \%}\right)$. Las variables categóricas fueron descritas en porcentajes. Se utilizó test de Fisher, $\chi^{2}, t$ de student y Wilcoxon Rank-Sum según característica y distribución de las variables. Para el análisis estadístico analítico se empleó el programa Stata ${ }^{\mathrm{R}} 14$, se consideró estadísticamente significativo un $\mathrm{p}<0,05$, se realizaron análisis uni y multivariado de los factores pronósticos calculando el odds ratio (OR) con un intervalo de confianza (IC) de 95\%. Para las curvas de sobrevida se empleó el método de Kaplan-Meier en el programa Prism $7^{\mathrm{MR}}$. En la creación de curvas ROC se empleó el programa Stata $^{\mathrm{R}} 14$.

\section{Seguimiento}

Considerando los criterios de exclusión, el presente estudio posee un $100 \%$ de seguimiento. El llenado de la base de datos se realiza en forma prospectiva con cada paciente, mientras que la actualización de la sobrevida se lleva a cabo en forma anual 
(mes de febrero), con la base de datos de nuestro hospital y del Registro Civil de Chile.

\section{Resultados}

Se incluyeron un total de 284 pacientes, de los cuales un $65,4 \%$ fueron hombres, la edad media fue 64,5 años ( $\pm 12,7$ DS). Un $69,7 \%$ de los pacientes presentaron comorbilidades siendo la hipertensión, el tabaquismo y la diabetes las más frecuentes, con un $38,3 \%, 34,8 \%$ y $17,6 \%$ respectivamente (Tabla 1). Según la clasificación $\mathrm{ASA}^{12}$, un $21,1 \%$ fueron ASA I, 70,7\% fueron ASA II y 8,0\% fueron ASA III. Un $24,6 \%$ de los pacientes presentaban familiares de primer grado con cáncer gástrico.

Tabla 1. Comorbilidades de la población global de pacientes (n: 284)

\begin{tabular}{|lcc|}
\hline & $\mathbf{n}$ & $\mathbf{\%}$ \\
Pacientes con comorbilidades & 198 & 69,7 \\
HTA & 109 & 38,3 \\
Tabaquismo & 99 & 34,8 \\
DM2 & 50 & 17,6 \\
DLP & 21 & 7,1 \\
IAM & 16 & 5,4 \\
Hipotiroidismo & 7 & 2,4 \\
ACV & 4 & 1,3 \\
ICC & 2 & 0,7 \\
Valvulopatías & 2 & 0,7 \\
\hline
\end{tabular}

HTA: Hipertensión arterial, DM2: Diabetes mellitus 2, DLP: Dislipidemia, IAM: Infarto agudo al miocardio, ACV: Accidente cerebrovascular, ICC: Insuficiencia cardíaca congestiva.

Figura 1. Órganos adicionales resecados en las gastrectomías ampliadas (n: 22).
En relación a la forma clínica de presentación un $55,9 \%$ presentaron epigastralgia, $10,5 \%$ disfagia y $10,1 \%$ saciedad precoz. La baja de peso se identificó en un $45,7 \%$ con una mediana de pérdida de $8 \mathrm{~kg}$ $(2-35 \mathrm{~kg})$. La anemia (HTO $<35 \%)$ estuvo presente en un $30,9 \%$, mientras que la desnutrición proteica (albúmina $<3,5 \mathrm{mg} / \mathrm{dl}$ ) en un $12,6 \%$.

Respecto a la técnica quirúrgica, $72,5 \%$ de los pacientes requirieron una gastrectomía total, $19,7 \%$ un subtotal y 7,8\% una gastrectomía ampliada. El detalle de los órganos extraídos adicionalmente se detalla en la Figura 1. La linfadenectomía practicada fue un D1 alfa o beta en un $14,7 \%$ y un D2 en un $85,2 \%$. La cosecha linfononodal global media fueron 30 linfonodos $\left(\mathrm{IC}_{25-75 \%}\right.$ : 22-41). El número de los ganglios cosechados en linfadenectomías D1 (alfa y beta) corresponde a 28 (IC $\left._{25 \%-75 \%}: 19-32,5\right)$, mientras que en D2 es 33 $\left(\mathrm{IC}_{25 \%-75 \%}: 24-44\right)$. El tiempo operatorio promedio fue de $208 \mathrm{~min}( \pm 63,8 \mathrm{DS})$. El $100 \%$ de la muestra fueron resecciones $\mathrm{R} 0$.

La estadía hospitalaria media de los pacientes fue de 9 días ( $\mathrm{IC}_{25-75 \%}$ : 7-11). La morbilidad quirúrgica posoperatoria correspondió a $17,2 \%$, mientras que la mortalidad quirúrgica fue de 5 pacientes (atribuidas a 3 filtraciones de esofagoyeyuno anastomosis, 1 neumonía asociada a ventilación mecánica y una fístula de muñón duodenal) lo que representa un 1,7\% (Tabla 2).

El estudio histopatológico identificó que un $58,4 \%$ de los tumores fueron de tipo intestinal,

Tabla 2. Morbilidad y mortalidad posoperatoria (n: 284)

\begin{tabular}{|lcl|}
\hline & n & \% \\
\hline $\begin{array}{l}\text { 1. Mortalidad } \\
\quad \text { Operatoria hasta los 30 días }\end{array}$ & 5 & 1,7 \\
2. Morbilidad quirúrgica & & \\
$\quad$ Colección intraabdominal & 24 & 8,4 \\
Fístula EY & 23 & 8 \\
Fístula de muñón duodenal & 10 & 3,5 \\
Infección herida operatoria & 8 & 2,8 \\
Obstrucción intestinal & 7 & 2,4 \\
Hemoperitoneo & 4 & 1,4 \\
Fístula pancreática tipo A & 1 & 0,3 \\
3. Morbilidad médica & & \\
$\quad$ Respiratorias & 22 & 7,7 \\
Cardiovasculares & 13 & 4,5 \\
Infecciosas médicas & 16 & 5,6 \\
\hline
\end{tabular}

SDRA: Síndrome de distrés respiratorio del adulto, TEP: Tromboembolismo pulmonar, ITU: infección del tracto urinario, ICC: insuficiencia cardíaca congestiva, IAM: infarto agudo de miocardio. 
mientras que el 36,6\% son de tipo difuso (indiferenciados o con células en anillo de sello) y un $4,9 \%$ fueron mixtos. Un $25 \%$ de la muestra corresponde a cánceres incipientes y un $75 \%$ a avanzados. Los pacientes con células en anillo de sello representan un $24,2 \%$ de la muestra total. La permeación linfovascular se identificó en un 50,3\% de la muestra, mientras que el compromiso perineural en un $41,5 \%$. La media de linfonodos comprometidos por paciente fue $2\left(\mathrm{IC}_{25-75 \%}: 0-9\right)$ y la media del índice ganglionar de toda la muestra fue $7 \%\left(\mathrm{IC}_{25-75 \%}: 0-31,2\right)$, lo que corresponde a una clasificación de LNR 1. Las Tablas 3 y 4 muestran la clasificación TNM y estadio de los pacientes.

La sobrevida global media fue de 69,9 meses (intervalo entre 1 y 158 meses, con DS $\pm 47,9$ ). La tasa de pacientes con una SVg5 fue de 56,9\%, de este grupo un 40,2\% fueron cánceres incipientes y un $59,8 \%$ avanzados, la SVg5 de estos fue 90,1\% y $44,6 \%$ respectivamente. Las curvas de sobrevida según clasificación $\mathrm{T}$ y $\mathrm{N}$ se detallan en la Figura 2, ambas gráficas muestras diferencias estadísticamente significativas entre grupos con un $\mathrm{p}<0,0001$ para cada una.

La sobrevida global mayor a 10 años de la población que completó el seguimiento entre 2004 hasta 2007 (133 pacientes), fue de 53,4\%, este tipo de sobrevida en cánceres incipientes $(27,1 \%$ de dicha cohorte) fue $88,8 \%$, mientras que en cánceres avanzados (72,9\% de dicha cohorte) fue $40,2 \%$.

Al comparar la evolución de la sobrevida entre pacientes con más y menos de 5 años de supervivencia, se objetiva que, dentro del grupo de pacientes con sobrevida exclusivamente menor a 5 años, un $50,7 \%$ fallece antes de los 2 años, mientras que la población de pacientes con sobrevida exclusivamen-
Tabla 3. Clasificación T y N (n: 284)

\begin{tabular}{|lll|}
\hline \multicolumn{1}{|c|}{$\mathbf{n}$} & $\mathbf{\%}$ \\
Clasificación T & & \\
T1 & 71 & 25 \\
T2 & 31 & 10,9 \\
T3 & 73 & 25,7 \\
T4 & 109 & 38,3 \\
Clasificación N & & \\
N0 & 114 & 40,1 \\
N1 & 33 & 11,6 \\
N2 & 39 & 13,7 \\
N3a & 55 & 19,3 \\
N3b & 43 & 15,1 \\
\hline
\end{tabular}

Clasificación TNM según la 7ª ed. de la AJCC de 2010.

Tabla 4. Estadios TNM (n: 284)

\begin{tabular}{|ccc|}
\hline Estadio & $\mathbf{n}$ & $\mathbf{\%}$ \\
\hline IA & 67 & 23 \\
\hline IB & 14 & 4,9 \\
\hline IIA & 27 & 9,5 \\
\hline IIB & 34 & 11,9 \\
\hline IIIA & 31 & 10,9 \\
\hline IIIB & 51 & 17,9 \\
\hline IIIC & 60 & 21,1 \\
\hline
\end{tabular}

Clasificación por estadio según la $7^{\mathrm{a}}$ ed. de la AJCC de 2010.

te mayor a 5 años presenta sólo una caída de 17,2\% entre el $5^{\circ}$ y $10^{\circ}$ año posoperatorio (Figura 3 ).

La sobrevida global según el índice linfondal de nuestra población estudiada se refleja en la Figura 4.
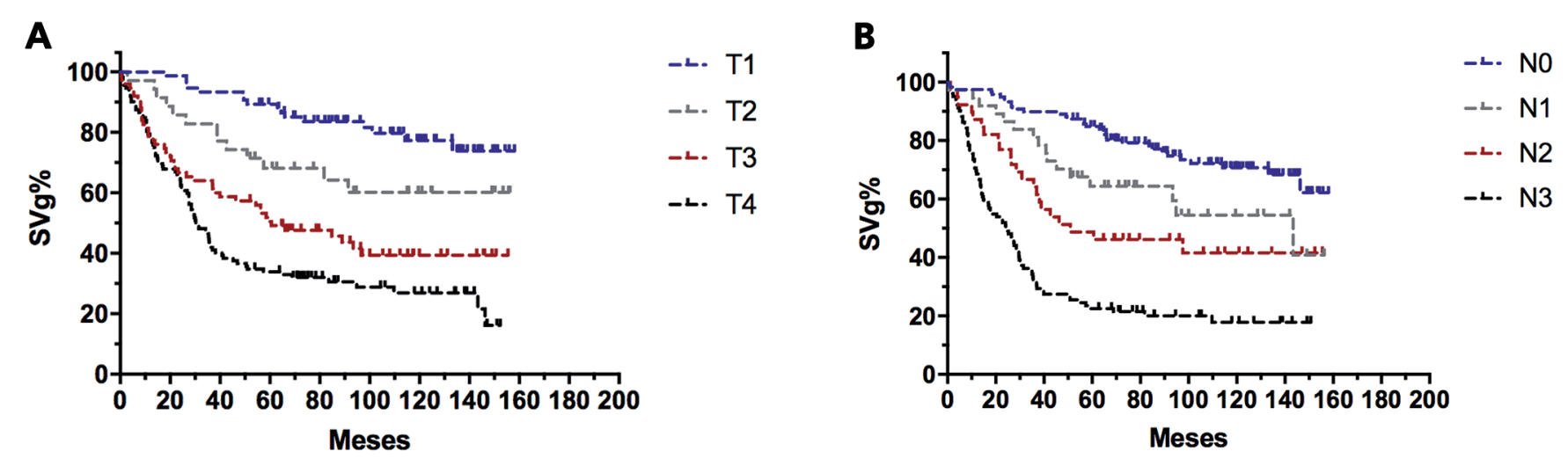

Figura 2. Curvas de sobrevida global según clasificación T y N. A) Sobrevida según T y B) Sobrevida según N. SVg\%: porcentaje de sobrevida global.

-ᄂ. $\mathrm{T} 1$

ㄴ. $\mathrm{T} 2$

ㄴ. $\mathrm{T} 3$

ㄴ. $\mathrm{T} 4$ 


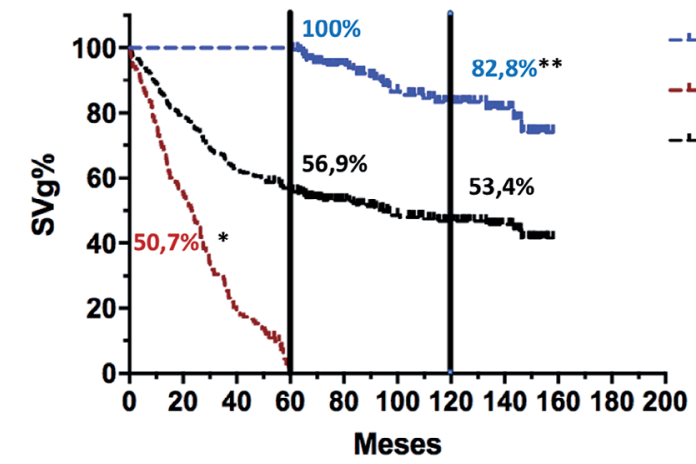

Figura 3. Curvas de sobrevida global diferenciando paciente con sobrevida global mayor y menor a 5 años. SVg $>5$ años: sobrevida global mayor a 5 años, $S V g<5$ años: sobrevida global menor a 5 años, SVg\%: porcentaje de sobrevida global. *50,7\% de los pacientes con sobrevida menor a 5 años fallece antes de los 24 meses. ${ }^{* *}$ Sobrevida a 10 años del grupo de pacientes con supervivencia mayor de 5 años entre 2004 y 2007.
La diferencia observada alcanza una significancia estadística con un $\mathrm{p}<0,0001$.

$\mathrm{Al}$ analizar el índice $\mathrm{N}+/ \mathrm{T}$, se identifica una diferencia estadísticamente significativa en la sobrevida global alejada de todos los subgrupos $(\mathrm{N}+/ \mathrm{T}$ A, $\mathrm{N}+/ \mathrm{T}$ B y N+/T C) con un $\mathrm{p}<0,0001$ (Figura 5).

Al realizar el estudio comparativo univariado entre pacientes con menos o más de 5 años de sobrevida global, se obtienen los resultados expuestos en la Tabla 5. En este tipo de análisis, el índice $\mathrm{N}+/ \mathrm{T}$ logra discriminar ambos grupos de manera estadísticamente significativa en cada una de sus divisiones $(\mathrm{N}+/ \mathrm{T}$ A, $\mathrm{N}+/ \mathrm{T}$ B y N+/T C). Los pacientes $\mathrm{N}+/ \mathrm{T}$ A presentan un $p<0,0001$. OR: 0,12 (IC 95\%: 0,07$0,21)$ ], mientras que los pacientes $\mathrm{N}+/ \mathrm{T} \mathrm{C}$ presentan un $\mathrm{p}<0,0001$. OR: 6,45 (IC 95\%: 3,42-12,09)]. (Tabla 4).

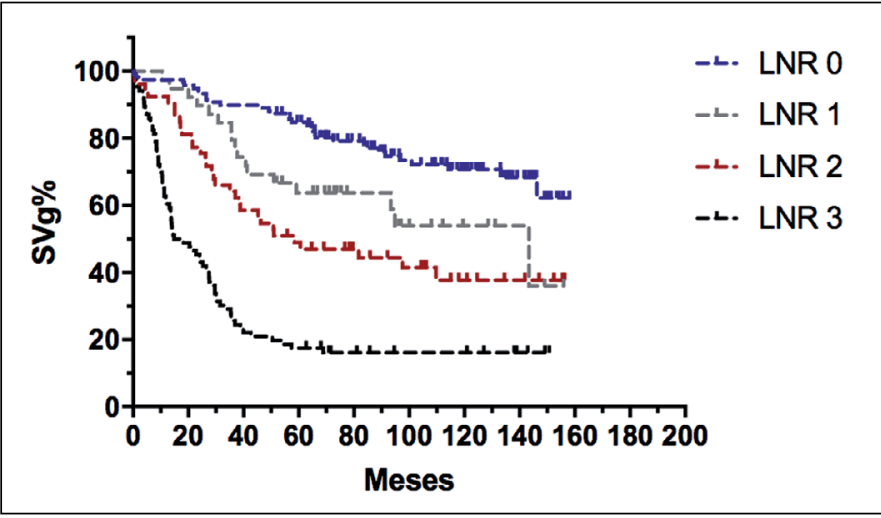

Figura 4. Curvas de sobrevida global alejada según grupos de LNR. SVg\%: porcentaje de sobrevida global.

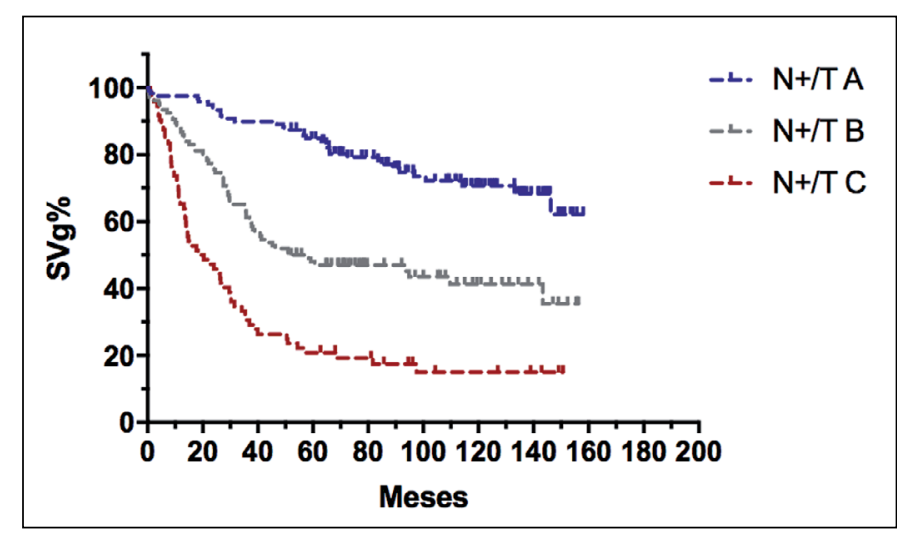

Figura 5. Curvas de sobrevida global alejada según grupos de índice $N+/ \mathrm{T}$. SVg\%: porcentaje de sobrevida global.

Tabla 5. Análisis univariado de factores pronóstico de sobrevida alejada (n: 279*)

\begin{tabular}{|c|c|c|c|c|c|c|}
\hline \multirow[t]{2}{*}{ Variable } & \multicolumn{2}{|c|}{$\mathrm{SVg}<5$ años } & \multicolumn{2}{|c|}{$\mathrm{SVg}>5$ años } & \multicolumn{2}{|c|}{ Análisis univariado } \\
\hline & n 120 & $\%$ & n 159 & $\%$ & p & OR (IC 95\%) \\
\hline \multicolumn{7}{|l|}{ Sexo } \\
\hline Hombres & 89 & 73,7 & 94 & 59,1 & 0,018 & $1,98(1,1-3,3)$ \\
\hline Mujeres & 31 & 26,2 & 65 & 40,8 & & \\
\hline \multicolumn{7}{|l|}{ Edad } \\
\hline Análisis como variable continua** & 63,5 & & 60,8 & & 0,002 & $1,02(1,00-1,04)$ \\
\hline \multicolumn{7}{|l|}{ ASA } \\
\hline I & 23 & 19,4 & 37 & 23 & 0,4 & \\
\hline II-III & 97 & 80,5 & 122 & 76,7 & & \\
\hline \multicolumn{7}{|l|}{ Comorbilidades } \\
\hline Tabaquismo & 53 & 44,9 & 76 & 47,7 & 0,6 & \\
\hline HTA & 48 & 39,8 & 57 & 35,8 & 0,5 & \\
\hline DM2 & 27 & 22 & 14 & 8,8 & 0,001 & $3,00(1,4-6,0)$ \\
\hline
\end{tabular}




\section{Continuación Tabla 5}

\begin{tabular}{|c|c|c|c|c|c|c|}
\hline \multirow[t]{2}{*}{ Variable } & \multicolumn{2}{|c|}{ SVg $<5$ años } & \multicolumn{2}{|c|}{ SVg $>5$ años } & \multicolumn{2}{|c|}{ Análisis univariado } \\
\hline & n 120 & $\%$ & n 159 & $\%$ & $\mathbf{p}$ & OR (IC 95\%) \\
\hline \multicolumn{7}{|l|}{ Clínica } \\
\hline Epigastralgia & 61 & 50,8 & 98 & 61,6 & 0,08 & \\
\hline Disfagia & 18 & 15,2 & 11 & 6,9 & 0,046 & $2,37(1,0-5,1)$ \\
\hline \multicolumn{7}{|l|}{ Laboratorio } \\
\hline HTO $<35 \%$ & 35 & 29,6 & 49 & 30,8 & 0,7 & \\
\hline \multicolumn{7}{|l|}{ Quirúrgico } \\
\hline Gastrectomía subtotal & 16 & 13,5 & 39 & 24,5 & 0,02 & $0,47(0,2-0,8)$ \\
\hline Gastrectomía total & 96 & 79,6 & 106 & 66,6 & & \\
\hline Gastrectomía ampliada & 8 & 6,7 & 14 & 8,8 & & \\
\hline \multicolumn{7}{|l|}{ Morbilidad quirúrgica } \\
\hline Fístula anastomótica & 10 & 8,4 & 13 & 8,1 & $>0,9$ & \\
\hline Neumonía & 5 & 2,5 & 3 & 1,8 & 0,2 & \\
\hline Arritmia & 1 & 0,8 & 7 & 4,4 & 0,1 & \\
\hline \multicolumn{7}{|l|}{ Histología } \\
\hline Células anillo de sello & 41 & 34,7 & 29 & 18,2 & 0,003 & $2,2 \quad(1,3-3,9)$ \\
\hline Permeación linfovascular & 83 & 68,6 & 59 & 37,1 & $<0,0001$ & $3,8 \quad(2,2-6,3)$ \\
\hline Compromiso perineural & 71 & 59,3 & 43 & 27 & 0,0005 & $2,5 \quad(1,5-4,3)$ \\
\hline \multicolumn{7}{|l|}{$\mathrm{T}$} \\
\hline 1 & 7 & 5,9 & 64 & 40,2 & $<0,0001$ & $0,09(0,03-0,21)$ \\
\hline 2 & 19 & 16,1 & 21 & 13,2 & & \\
\hline 3 & 33 & 27,9 & 37 & 23,2 & & \\
\hline 4 & 70 & 57,6 & 37 & 23,2 & & \\
\hline \multicolumn{7}{|l|}{ Estadio } \\
\hline IIA & 3 & 2,5 & 23 & 14,4 & & \\
\hline IIB & 14 & 11 & 20 & 12,5 & & \\
\hline IIIA & 13 & 11 & 17 & 10,6 & & \\
\hline IIIB & 36 & 29,6 & 13 & 8,1 & & \\
\hline IIIC & 45 & 38,1 & 14 & 8,8 & & \\
\hline \multicolumn{7}{|l|}{ Índice linfonodal } \\
\hline LNR $0(0 \%)$ & 16 & 12,7 & 97 & 61 & $<0,0001$ & $0,08(0,04-0,16)$ \\
\hline LNR 1 (1-9\%) & 14 & 11,8 & 22 & 13,8 & & \\
\hline LNR $2(10-25 \%)$ & 25 & 21,1 & 25 & 15,7 & & \\
\hline LNR 3 (> 25\%) & 65 & 54,2 & 15 & 9,4 & & \\
\hline \multicolumn{7}{|l|}{ Índice $\mathrm{N}+/ \mathrm{T}$} \\
\hline $\mathrm{A}(0)$ & 20 & 16,1 & 97 & 61 & $<0,0001$ & $0,12(0,07-0,23)$ \\
\hline $\mathrm{B}(0,1-3)$ & 52 & 43,2 & 48 & 30,1 & & \\
\hline$C(>3)$ & 48 & 40,6 & 14 & 8,8 & & \\
\hline
\end{tabular}

IC: Intervalo de confianza, OR: Odds ratio, SVg $>5$ años: sobrevida global mayor a 5 años, SVg $<5$ años: sobrevida global menor a 5 años. $*$ Se excluyeron los 5 pacientes que presentaron mortalidad quirúrgica, **El análisis de edad se realizó considerándola como variable continua, las edades expresadas en las columnas son los promedios de edad de los pacientes con menos y más de 5 años de sobrevida. 
El análisis multivariado de los factores pronósticos está representado en la Tabla 6 , donde se evidencia que las variables que persisten siendo significativas son: índice $\mathrm{N}+/ \mathrm{T}$, LNR, edad, permeación linfovascular, clasificación $\mathrm{T}$, clasificación $\mathrm{N}$ y estadio TNM.

La curva ROC del índice $\mathrm{N}+/ \mathrm{T}$ y las curvas comparativas entre las variables predictoras $\mathrm{T}$ y LNR se representa en la Figura 6. El área bajo la curva de $\mathrm{N}+/ \mathrm{T}$ es 0,789 y la diferencia apreciada entre las curvas de las tres variables comparadas no alcanza una significancia estadística, con un $\mathrm{p}=0,96$.

Tabla 6. Análisis multivariado de factores pronóstico de sobrevida alejada

\begin{tabular}{|llc|}
\hline Variable & \multicolumn{2}{c|}{ Análisis multivariado } \\
Edad & p & OR (IC 95\%) \\
Célula anillo de sello & 0,008 & $1,03(1,00-1,06)$ \\
Permeación linfovascular & 0,011 & $1,69(1,13-2,55)$ \\
Clasificación T & 0,0001 & $2,19(1,49-3,23)$ \\
Clasificación N & 0,03 & $3,14(1,10-8,93)$ \\
Estadio TNM & 0,001 & $1,06(1,02-1,10)$ \\
LNR & 0,0001 & $9,13(3,27-25,4)$ \\
N+/T & 0,0001 & $5,18(1,04-15,6)$ \\
\hline
\end{tabular}

IC: Intervalo de confianza, OR: Odds ratio. LNR: Índice linfonodal.

\section{Discusión}

Los resultados principales de este estudio sugieren que:

1) La población de pacientes estudiados, poseen características demográficas comparables a otras publicaciones.

2) Los resultados quirúrgicos de morbilidad y mortalidad posoperatorio son aceptables según estándares internacionales.

3) Los resultados oncológicos cumplen modelos de calidad en el manejo moderno del cáncer gástrico.

4) En el grupo de pacientes con sobrevida menor a 5 años, la mayoría fallecen antes de los 24 meses, en su mayoría por progresión de enfermedad.

5) Existen distintas variables que predicen de manera significativa la población susceptible de alcanzar una sobrevida alejada posoperatoria.

6) El índice $\mathrm{N}+/ \mathrm{T}$ es una herramienta que puede ser útil en la predicción de sobrevida mayor a 5 años en pacientes operados de adenocarcinoma gástrico con intención curativa.

La presentación y tratamiento del cáncer gástrico ha cambiado en los últimos 40 años. La frecuencia de tumores distales disminuyó de $64 \%$ a $25 \%$, la proporción de tipo intestinal:difusa pasó de 3-4:1 a casi $1: 1$, la tasa de resecabilidad ha aumentado de $48 \%$ a $85 \%$ ( $p<0,001)$, un $75 \%$ de los casos son gastrectomías totales y la mortalidad quirúrgica ha disminuido de $25 \%$ a $0,8 \%(\mathrm{p}<0,0001)^{1,13}$.

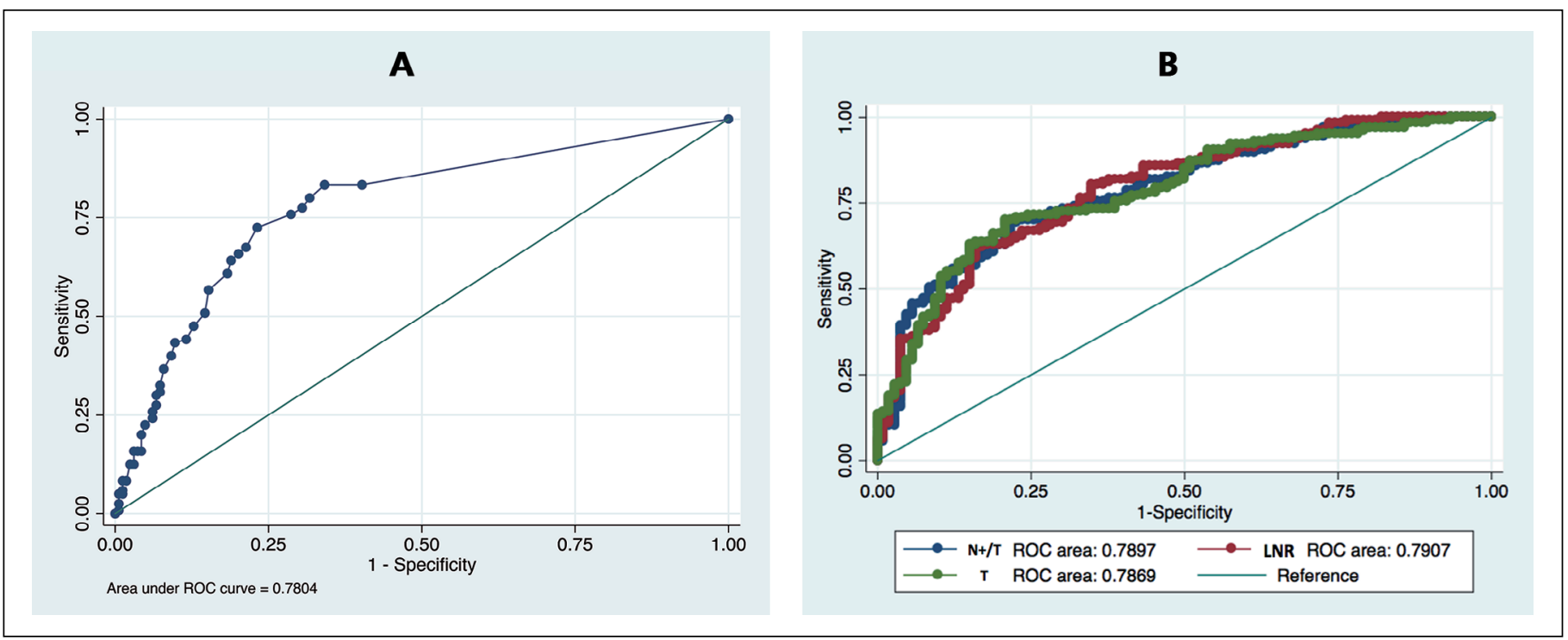

Figura 6. Curvas ROC. A) curva $N+/ T$ con un área bajo la curva igual a 0,78 ; B) curva de $N+/ T$ comparativa con $T$ y LNR con áreas bajo la curva iguales a 0,78 y 0,79 respectivamente. 
Los cambios epidemiológicos presentan distintas explicaciones ${ }^{13}$, mientras que los mejores resultados posoperatorios se han debido principalmente a 2 factores: "factor primario y factor secundario", el primero hace mención al rol de cirujano y su equipo quirúrgico especializado en esta patología, mientras que el factor secundario es representado por los avances de medicamentos y las unidades de apoyo como cuidados intensivos, nutrición médica, kinesioterapias, radiología intervencional, entre otras $^{14}$.

La serie estudiada en el presente artículo refleja parte de estos cambios. Es así como un 40\% de los casos son lesiones difusas, $2 / 3$ de la población requirieron de una gastrectomía total (esta cifra aumenta si se consideran el grupo de pacientes con gastrectomía total ampliada, del cual el 100\% fueron totales), la morbilidad posoperatoria es de $25,9 \%$ y la mortalidad quirúrgica es $1,7 \%$, estando estas cifras dentro de los estándares de calidad expuestos en el último consenso panamericano de cáncer gástrico ${ }^{15}$.

La SVg5 de los pacientes en el presente estudio es de $56,9 \%$, este valor es comparable a otras publicaciones ${ }^{16}$. Al analizar los casos con sobrevida menor de 5 años cerca de un 50\% fallece antes de los 24 meses, en su mayoría por progresión de enfermedad, lo cual se ha documentado en otros reportes ${ }^{4,17,18}$. Un aspecto interesante es que la curva de sobrevida de la población con supervivencia mayor a 5 años, tiende a aplanarse entre el $5^{\circ}$ y $10^{\circ}$ año de seguimiento posoperatorio, lo que objetiva que el principal período de mortalidad en este grupo de pacientes, es en los primeros 2 años posoperatorio.

En relación a factores pronósticos de sobrevida alejada, se han diseñado guías que permiten tomar decisiones en relación a estrategias terapéuticas para optimizar el pronóstico oncológico de estos pacientes, es así como la clasificación TNM ha permitido pautar el pronóstico y manejo de estos pacientes, su última actualización es del año 2010 (AJCC, Chicago, Illinois), mientras que las últimas pautas del NCCN son de $2017^{5}$.

La eficacia pronóstica de la clasificación TNM y sus estadios es conocida y ha sido analizada por distintos grupos de trabajo internacionales ${ }^{7,19,20}$ y nacionales ${ }^{16,21,22}$.

En los últimos años se han analizado distintos factores complementarios predictores de sobrevida alejada.

\section{Género}

La evidencia en relación al impacto del género en la sobrevida de pacientes con cáncer gástrico es controversial, si bien existen artículos que defienden una significancia estadística ${ }^{23,24}$, hay evidencia que se contrapone ${ }^{25,26}$.

Los resultados de esta publicación sugieren una asociación entre el sexo femenino y sobrevida alejada con un $\mathrm{p}=0,014$ [OR: 1,88 (IC 95\%: 1,15$3,07)]$. Sin embargo, en el análisis multivariado no se demostró significancia estadística.

\section{Edad}

El rol de la edad en el pronóstico de los pacientes sometidos a gastrectomía por cáncer ha sido estudiado por distintos autores $23,27,28$.

En esta serie no se logró identificar impacto pronóstico de la edad en la evolución de los pacientes tomando como margen los 70 años. Sin embargo, al evaluar la edad como variable continua, se determinó que a mayor edad existe peor pronóstico oncológico, tanto en análisis uni como multivariado con un $\mathrm{p}=0,008$.

\section{Estado nutricional}

El estado nutricional ha sido ampliamente estudiado por distintos autores tanto en el período preoperatorio como posoperatorio, es así como Liu analiza el impacto del IMC, la albúmina y triglicéridos preoperatorios, logrando identificar significancia en análisis multivariado de estos factores en el pronóstico de sobrevida ${ }^{29}$. Jun también estudió el impacto en supervivencia del IMC preoperatorio en una población de 211 cánceres gástricos avanzados estadio II-III sometidos a cirugía curativa y quimioterapia adyuvante, sus hallazgos apoyan un factor protector de un IMC $\geq 23 \mathrm{~kg} / \mathrm{mt}^{2}$, aunque no logra demostrar impacto en recurrencia ${ }^{30}$.Un factor recientemente descrito es la sarcopenia en el pronóstico de estos pacientes. Zheng analizó una población cercana a 1.000 cánceres gástricos sometidos a gastrectomía radical, en su estudio logró establecer cómo la sarcopenia, definida a través del índice musculoesquelético por tomografía computada, impacta negativamente el pronóstico de los pacientes, con una sobrevida a 3 años de $55 \%$, en comparación a la población sin sarcopenia con una sobrevida de $75 \%$. Además, ideó un score pronóstico combinando cT, cN y sarcopenia, demostrando una sobrevida a 3 años de $89 \%$ en la población de bajo riesgo y de $54,8 \%$ para el grupo de alto riesgo $(\mathrm{p}<0,001)^{26}$. En el análisis de la evolución nutricional posoperatoria, un estudio coreano con más de 1.900 pacientes revisó en forma retrospectiva aquellos pacientes con cáncer gástrico sometidos a gastrectomía, este estudio concluyó que un IMC > $25 \mathrm{~kg} / \mathrm{mt}^{2}$ al año posoperatorio representa un factor 
protector en sobrevida global en comparación a la población normopeso, alcanzando un $49 \%$ de reducción de mortalidad (HR 0,51; IC 95\%: 0,26$0,98)^{31}$.

\section{Células tumorales circulantes}

Lee logró mostrar una mejor sobrevida libre de enfermedad en aquellos pacientes con $\leq 5$ células tumorales circulantes en 7,5 $\mathrm{ml}$ de sangre periférica con un $\mathrm{p}=0,034^{32}$.

Sin embargo, una desventaja de este marcador es su costo, es así como esta realidad existe en modelos experimentales, ya que resulta poco implementable, por ahora, como método de estudio para políticas públicas.

\section{Tipo de gastrectomía}

Generalmente es aceptado que aquellos tumores gástricos distales poseen mejor pronóstico que los cardiales, esto se ha atribuido a que en la cara posterior del área subcardial existe una región gástrica retroperitonal sin cobertura serosa, la cual pudiese predisponer a una invasión tumoral de la grasa perigástrica, y por ende poseer un estadio mayor, en forma más precoz $z^{33,34}$. De esta forma, existen reportes que atribuyen a las gastrectomías subtotales un factor protector en relación a sobrevida alejada ${ }^{7,35-42}$. Esta evidencia es concordante con lo expuesto en el presente estudio donde las gastrectomías subtotales presentaron un factor protector estadísticamente significativo para sobrevida alejada con un $\mathrm{p}=0,01$. Sin embargo, esta asociación se pierde luego del análisis multivariado.

\section{Pérdidas sanguíneas}

Lian estudió una población de 845 cánceres gástricos estadios I-III sometidos a gastrectomía curativa. Concluyó que existe un impacto en sobrevida inversamente proporcional a las pérdidas hemáticas intraoperatorias, pérdidas menores a 200 cc presentan una sobrevida alejada de $51 \%$, mientras que pérdidas de 200-400 cc tienen 39\% y aquellas mayores de 400 cc sólo $23 \%{ }^{25}$.

\section{Histología}

Históricamente se ha evaluado el impacto de la diferenciación tumoral y el compromiso linfovascular en la sobrevida global de los pacientes. Al igual que lo objetivado en este trabajo, donde la presencia de células en anillo de sello, la permeación linfovascular y el compromiso perineural lograron demostrar significancia pronóstica. Un estudio chino de 323 pacientes operados en forma curativa mostró en el análisis multivariado que el grado de diferenciación, el compromiso vascular y las metástasis linfáticas son herramientas pronósticas para determinar sobrevida libre de enfermedad, con un hazard ratio (HR) de 3,5, 3,4 y 4,2 respectivamente; al evaluar estos factores en el pronóstico de sobrevida global y de metástasis hematógena, sólo la diferenciación celular se mantuvo estadísticamente significativa con un HR de 1,6 y 5,623.

\section{Nomograma}

Una manera de integrar el valor pronóstico de distintos factores es el desarrollo de nomogramas, así un estudio norteamericano multicéntrico con más de 1.600 pacientes desarrolló un nomograma que combina: sexo, edad al momento de cirugía, localización tumoral, clasificación de Lauren, diámetro tumoral, T y N. Al seguir la pauta de su estudio se obtiene un índice de concordancia de 0,77 , logrando mejorar la capacidad de predicción de sobrevida específica relacionada a enfermedad a 5 años ${ }^{24}$,

\section{Índice linfonodal}

El índice linfonodal o LNR (lymph node ratio) compara la razón entre los linfonodos comprometidos y la cosecha linfonodal total. Se han estudiado distintos puntos de corte en subgrupos, Sin embargo, para el presente estudio se definieron los límites propuestos por un estudio chino basado en 710 pacientes sometidos a gastrectomía $\mathrm{R} 0^{7}$, en éste se definió los márgenes LNR en 4 categorías: $0(0 \%)$, $1(1-9 \%), 2(10-25 \%), 3(>25 \%)$. Los resultados del análisis multivariado mostraron que el índice linfonodal, a diferencia de la clasificación TNM empleada, lograba predecir sobrevida de manera independiente.

Otros autores también han logrado demostrar el impacto del LNR en la sobrevida de los pacientes ${ }^{19,21,27,40}$. Estas experiencias se reproducen en el presente estudio, el LNR logró mostrar significancia estadística en el análisis multivariado, con un gran poder pronóstico en el subgrupo LNR2, con un $\mathrm{p}<0,0001$; OR: 10,17 (IC 95\% 5,37-18,8), en su contraparte el LNR0 ofrece un factor protector para sobrevida mayor a 5 años, con un $\mathrm{p}<0,0001$; OR: 0,12 (IC 95\% 0,07-0,21).

\section{Índice $N+/ T$}

Respecto al índice $\mathrm{N}+/ \mathrm{T}$ resulta lógico pensar que la potencialidad metastásica linfonodal de un tumor dependiendo de su grado de invasión pudiese predecir en forma fidedigna el pronóstico de los pacientes. De esta forma se ideó la razón entre los linfonodos comprometidos y la clasificación $\mathrm{T}$, logrando demostrarse una diferenciación significativa 
entre las curvas de sobrevida global de los distintos subgrupos con un $\mathrm{p}<0,0001$ (Figura 5). Además, se objetivó una asociación pronóstica potente tanto en el análisis uni como multivariado para predecir sobrevida alejada a más de 5 años, con un $\mathrm{p}<0,0001$, 0,008 y $<0,0001$, para los subgrupos A, B y C respectivamente. El análisis de curvas ROC (Figura 6) muestra cómo el índice $\mathrm{N}+/ \mathrm{T}$ posee un área bajo la curva de 0,789 , sin diferencia estadísticamente significativa de los factores T y LNR con un $\mathrm{p}=0,96$. Si bien en el grupo de pacientes con sobrevida mayor a 5 años no se identificó diferencias pronósticas estadísticamente significativas en la evaluación de $\mathrm{T}, \mathrm{N}$ y $\mathrm{N}+/ \mathrm{T}$, es posible que con una población mayor de pacientes estudiados se habría alcanzado la significancia. Sin embargo, existe un reporte hecho por Moon ${ }^{20}$ quien en una cohorte de 500 pacientes con cáncer gástrico avanzado tratados con intención curativa, identifica que posterior a los 5 años de seguimiento, el TNM no logra predecir evolución excepto en estadios IV y sobre los 10 años de seguimiento ninguna de las variables estudiadas predijo evolución.

Las publicaciones nacionales enfocadas a pronóstico de supervivencia en cáncer gástrico se han centrado en distintos aspectos. En términos de SVg5 la primera publicación chilena fue realizada por Frenkel en $1954^{43}$, donde describió una SVg5 de $12,7 \%$. Veinte años después en 1974, Csendes reportó el primer estudio multicéntrico de SVg5 en cáncer gástrico en Chile. Dicha publicación objetivó una SVg5 de 19\% en los pacientes reseca$\operatorname{dos}^{44}$, desde entonces esta tasa se ha mejorado hasta cifras cercanas a $49 \%$ y $65 \%{ }^{21,22,35,55}$. Al evaluar exclusivamente los cánceres incipientes Csendes recientemente publicó una SVg5 de $88 \%$ y de $72 \%$ a 15 años ${ }^{45}$, mientras que Ceroni publicó una SVg5 de $98 \%$ y de $94,7 \%$ a 10 años $^{16}$ (Tabla 7).

En cuanto al estudio de variables pronósticas de esta sobrevida, la mayoría de las publicaciones se enfocan en variables similares, como la clasificación de Lauren, la profundidad de invasión tumoral, el compromiso linfonodal y el estadio oncológico según clasificación $\mathrm{TNM}^{4,16,21,22,35,36-39,44,46-51,55}$. Algunas publicaciones han estudiado el impacto de la gastrectomía subtotal ${ }^{35-39,55}$, menos publicaciones han estudiado el impacto de la presencia de células en anillo de sello ${ }^{36,53}$, el uso de adyuvancia ${ }^{48,52,54}$, complicaciones posoperatorias ${ }^{48}$ y el índice linfonodal $^{21}$. Las fortalezas de este trabajo son: a) tener una población estudiada numerosa y representativa de la realidad nacional e internacional; b) dicha población ha sido manejada siguiendo estándares internacionales de tratamiento del cáncer gástrico
Tabla 7. Sobrevida a 5 años reportada por autores chilenos en el tratamiento del cáncer gástrico con resecciones con intención curativa

\begin{tabular}{|c|c|c|c|}
\hline Autor & Año & $\mathbf{n}$ & SVg $>5$ años (\%) \\
\hline Csendes $^{44}$ & 1974 & 752 & 19 \\
\hline Csendes $^{51}$ & 1975 & 285 & 21,4 \\
\hline Burmeister $^{38}$ & 1986 & 70 & 45,7 \\
\hline Csendes $^{55}$ & 1993 & 233 & 60 \\
\hline Cenitagoya $^{4}$ & 1997 & 286 & 41,2 \\
\hline Venturelli35 & 1999 & 94 & 44,1 \\
\hline Stambuk $^{21}$ & 2006 & 108 & 48,9 \\
\hline García $^{22}$ & 2007 & 423 & 52 \\
\hline Butte $^{37 *}$ & 2008 & 105 & 90,5 \\
\hline Tapia $^{53}$ & 2010 & 451 & 51 \\
\hline Venturelli $^{36}$ & 2010 & 52 & 47 \\
\hline Ceroni $^{16 *}$ & 2011 & 161 & 98 \\
\hline Csendes $^{45} *$ & 2015 & 304 & 88 \\
\hline Norero $^{48}$ & 2016 & 580 & 39 \\
\hline Manterola ${ }^{49}$ & 2016 & 22 & 37 \\
\hline Figueroa & 2017 & 284 & 56,9 \\
\hline
\end{tabular}

*Publicaciones que analizan sólo cáncer gástrico incipiente.

con intención curativa por un número reducido de cirujanos expertos en este tema; c) analizar el mayor número de variables pronósticas de supervivencia alejada en cáncer gástrico reportado en la literatura nacional y d) aportar un nuevo índice predictor de sobrevida.

Las debilidades de esta investigación son: a) ser un estudio retrospectivo, sometido a los sesgos propios de dicha metodología; b) abarcar un período de tiempo donde existió un cambio de clasificación TNM (de $6^{\mathrm{a}}$ a $7^{\mathrm{a}}$ edición) lo cual impacta en la clasificación y posiblemente en el manejo de estos pacientes.

En conclusión, el presente estudio logra demostrar la presencia de distintos factores pronósticos de cáncer gástrico concordantes con evidencias internacionales. Los factores pronósticos independientes de sobrevida mayor a 5 años son: índice $\mathrm{N}+/ \mathrm{T}$, LNR, edad, permeación linfovascular, clasificación $\mathrm{T}$, clasificación $\mathrm{N}$ y estadio TNM. Concomitantemente se ha logrado aportar un nuevo cuociente pronóstico en la evaluación de pacientes con adenocarcinoma gástrico resecados con intención curativa, el índice $\mathrm{N}+/ \mathrm{T}$. 


\section{Responsabilidades éticas}

Protección de personas y animales. El autor declara que para esta investigación no se han realizado experimentos en seres humanos ni en animales.

Confidencialidad de los datos. El autor declara que han seguido los protocolos de su centro de trabajo sobre la publicación de datos de pacientes.

Derecho a la privacidad y consentimiento informado. El autor ha obtenido el consentimiento informado de los pacientes y/o sujetos referidos en el artículo. Este documento obra en poder del autor de correspondencia.

\section{Financiación}

El autor declara no haber recibido ninguna financiación para la realización de este trabajo.

\section{Conflicto de intereses}

El autor declara no tener ningún conflicto de intereses.

\section{Agradecimientos}

Prof. Dr. Attila Csendes por su tutoría en el desarrollo del estudio. Drs. Katya Carrillo y Stefan Danilla por su apoyo estadístico. EU. Solange Cortés por su trabajo en la mantención de la base de datos oncológica $\mathrm{HCUCH}$.

\section{Bibliografía}

1. Csendes A, Burdiles P, Braghetto I, Diaz JC, Maluenda F, Korn O, et al. Resecabilidad y mortalidad operatoria de la gastrectomía subtotal y total en paciente con cáncer gástrico avanzado entre 1969 y 2004. Rev Med Chile. 2006;134:426-32.

2. Armijo R, Detels R, Coulson A, Medina E, Orellana M, González A. Epidemiología del cáncer gástrico en Chile. Rev Med Chile. 1981;109:551-6.

3. Otaiza E, Lopetegui G, Csendes A. Operabilidad y resecabilidad del cáncer gástrico. Rev Med Valpo. 1969;22:228-30.

4. Cenitagoya G, Berghi C, Keniger J, Casanueva D. Cáncer gástrico. Sobrevida real a 5 años. Rev Chil Cir. 1997;49:62932.

5. NCCN Guidelines Version 1.2017, 03/21/17. Gastric Cancer. https://www. nccn.org/professionals/physician_gls/f guidelines.asp

6. Venturelli A, Díaz, JC, Avendaño R. Sobrevida del cáncer gástrico en Chile. En Csendes A, Burdiles P, Korn O. Cáncer Gástrico. Capítulo 29; editorial Mediterráneo, Santiago de Chile. $2^{\mathrm{a}}$ ed. 2006;279-85.

7. Alatengbaolide, Lin D, Li Y, Xu H, Chen J, Wang B, et al. Lymph Node Ratio Is an Independent Prognostic Factor in Gastric Cancer After Curative Resection (R0) Regardless of the Examined Number of Lymph Nodes. An J Clin Oncol. 2013;36:325-30
8. Guía Clínica diabetes mellitus tipo 2 . Serie guías clínicas MINSAL. Santiago, MINSAL, 2010.

9. Guía clínica AUGE. Examen medicina preventiva. Serie Guías clínicas MINSAL. Santiago: MINSAL, 2013.

10. Guía clínica AUGE. Hipotiroidismo en personas de 15 años y más. Serie Guías clínicas MINSAL. Santiago: MINSAL, 2013.

11. Guía clínica hipertensión arterial primaria o esencial en personas de 15 años y más. Santiago: MINSAL, 2010, Normas técnicas. Dislipidemias. Santiago: MINSAL, 2000

12. Tiret L, Hatton F, Desmonts JM, Vourch G. Prediction of outcome of anaesthesia in patients over 40 years: A multifactorial risk index. Stat Med. 1988;7:947-54.

13. Csendes A, Figueroa M. Situación del cáncer gástrico en el mundo y en Chile. Rev Chil Cir. 2017;69:502-7.

14. Csendes A, Braghetto I, Díaz JC, Castillo J, Rojas J, Cortés S. Morbilidad y mortalidad operatoria de la gastrectomía subtotal y total por cáncer gástrico 2004 a 2010. Parte I de un estudio prospectivo. Rev Chil Cir. 2011;63:583-8.

15. Consenso $3^{\text {er }}$ Congreso Panamericano de Cáncer Gástrico. 2016, Santiago de Chile.

16. Ceroni M, García C, Benavides C, Covacevich S, Rubilar P, Cid H, et al. Seguimiento mayor a 10 años de pacientes operados por cáncer gástrico incipiente. Rev Chil Cir. 2011;63:591-8.

17. Verlato G, Marrelli D, Accordini S,
Bencivenga M, Di Leo A, Marchet A, et al. Short-term and long-term risk factors in gastric cancer. World J Gastroenterol. 2015;21:6434-43.

18. Csendes A. ¿Recurrencia o progresión de cáncer? Rev Chil Cir. 2005;57:288-90.

19. Nakagawa M, Choi YY, An JY, Hong JH, Kim JW, Kim HI, et al. Staging for Remnant Gastric Cancer: The Metastatic Lymph Node Ratio vs. the UICC 7th Edition System. Ann Surg Oncol. 2016;23:4322-31.

20. Moon Y, Jeung H, Rha S, Yoo N, Roh J, Noh S, et al. Changing Patterns of Prognosticators During 15-Year FollowUp of Advanced Gastric Cancer after Radical Gastrectomy and Adjuvant Chemotherapy: A 15-Year Follow-Up Study at a Single Korean Institute. Ann Surg Oncol. 2007;14:2730-7.

21. Stambuk J. Resultados inmediatos y sobrevida alejada en cáncer gástrico: Estudio de 108 pacientes sometidos a gastrectomía total radical D2 con criterio R0. Rev Chil Cir. 2006;58:420-30.

22. García C, Benavides C, Apablaza S, Rubilar P, Covacevich R, Peñaloza P, et al. Resultados del tratamiento quirúrgico del cáncer gástrico. Análisis de 423 casos. Rev Med Chile. 2007;135:687-95.

23. Huang B, Wang Z, Xing C, Sun Z, Zhao $\mathrm{B}, \mathrm{Xu} \mathrm{H}$. Long-term survival results and prognostic factors of early gastric cancer. Exp Ther Med. 2011;2:1059-64.

24. Dikken J, Baser R, Gonen M, Kattan M, Shah M, Verheik M, et al. Conditional 
Probability of Survival Nomogram for 1-, 2-, and 3-Year Survivors After an R0 Resection for Gastric Cancer. Ann Surg Oncol. 2013;20:1623-30.

25. Lian Y, Gu H, Deng J, Wang B, Ding X, Wang $X$, et al. Impact of intraoperative blood loss on survival after curative resection for gastric cancer. World $\mathrm{J}$ Gastroenterol. 2013;17:5542-50.

26. Zheng Z, Lu J, Zheng C, Li P, Xie J, Wang J, et al. A Novel Prognostic Scoring System Based on Preoperative Sarcopenia Predicts the Long-Term Outcome for Patients After R0 Resection for Gastric Cancer: Experiences of a High-Volume Center. Ann Surg Oncol. 2017;24:1795803.

27. Posteraro B, Persiani R, Dall'Armi V, Biondi A, Arzani D, Sicoli F, et al. Prognostic factors and outcomes in Italian patients undergoing curative gastric cancer surgery. EJSO. 2014;40:345-51.

28. Jie, Chen J, Xu Y, Long Z, Zhou Y, Zhu H, et al. Impact of Age on the Prognosis of Operable Gastric Cancer Patients. An Analysis Based on SEER Database. Medicine 2016;95:e3944.

29. Liu BZ, Tao L, Chen YZ, Li XZ, Dong YL, Ma YJ, et al. Preoperative Body Mass Index, Blood Albumin and Triglycerides Predict Survival for Patients with Gastric Cancer. Plos ONE. 2016;11:e157401.

30. Jun DH, Kim BJ, Park JH, Kim JG, Chi KC, Park JM, et al. Preoperative Body Mass Index May Determine the Prognosis of Advanced Gastric Cancer. Nutr Cancer. 2016;68:1295-300.

31. Lee HH, Park JM, Song KY, Choi MG, Park CH. Survival impact of postoperative body mass index in gastric cancer patients undergoing gastrectomy. Eur J Cancer. 2016;52:129-37.

32. Ito $\mathrm{H}$, Sato J, Tsujino $\mathrm{Y}$, Yamaguchi $\mathrm{N}$, Kimura S, Gohda K, et al. Long-term prognostic impact of circulating tumor cells in gastric cancer patients. World $\mathrm{J}$ Gastroenterol. 2016;14:10232-41.

33. Siewert JR. Böttcher K, Stein HJ, Roder JD, Busch R. Problem of proximal third gastric carcinoma. World J Surg. 1995;19:523-31.

34. Testut L, Latarjet. Estómago, en:
Anatomía Humana. Salvat, Barcelona, $9^{\mathrm{a}}$ ed. Tomo IV, Capítulo I. 1971:184-187 y 207-8.

35. Venturelli A, Cardemil B, Díaz J, Avendaño R, Murúa A, Kuschel C, et al. Cirugía más quimioterapia en cáncer gástrico: sobrevida a 5 años. Rev Chil Cir. 1999;51:170-5.

36. Venturelli F, Venturelli A, Cárcamo M, Cárcamo C, Jara C, Felmer O, et al. Gastrectomía subtotal en el cáncer gástrico antral mal diferenciado e indiferenciado. Rev Chil Cir. 2010;62:240-5.

37. Butte J, Torres J, Viviani P, Duarte I, Crovari F, Guzmán S, et al. Sobrevida alejada de pacientes operados por cáncer gástrico incipiente. Rev Med Chile 2008;136:1424-30.

38. Burmeister R, Pepper J, Fernandez M. Sobrevida a largo plazo del cáncer gástrico. Rev Chil Cir. 1986;38:193-6.

39. Csendes A. Medina E. Sobrevida de pacientes con cáncer gástrico. III Sobrevida del cáncer avanzado según tipo de resección quirúrgica. Rev Med Chile. 1975;103:244-6.

40. Wang W, Li YF, Sun X, Chen YB, Li W, $\mathrm{Xu}$ DZ, et al. Prognosis of 980 patients with gastric cancer after surgical resection. Chinese Journal of Cancer. 2010;29:92330.

41. Bolt WJ, Devesa SS, Kneller RW, Fraumeni JF Jr. Rising incidence of adenocarcinoma of the esophagus and gastric cardia. JAMA. 1991;265:1287-9.

42. Craanen ME, Dekker W, Blok P, Ferwerda J, Tytgat GN. Time trends in gastric carcinoma: changing patterns of type and location. Am J Gastroenterol. 1992;87:572-9.

43. Frenkel A. Cáncer gástrico y gastrectomía total. Archivos Sociedad de Cirujanos. 1954;6:1597-618.

44. Csendes A, Medina E. Sobrevida de pacientes con cáncer gástrico. I Sobrevida global en casos operables y no operables. Rev Med Chile. 1974;102:837-840.

45. Csendes A, Díaz JC, Musleh M, Lanzarin E, Braghetto I, Zamorano M. Evolución durante 44 años de las características morfológicas y sobrevida a largo plazo de 304 pacientes con cáncer gástrico incipiente. Rev Chil Cir. 2015;67:175-80.

46. Pimentel F, Guzmán S, Llanos O, Rahmer A, Zúñiga A, Meissner A. Cáncer gástrico: resultados quirúrgicos y sobrevida. Rev Med Chile. 1988;116: 525-31.

47. Astete G, Lynch O, Madariaga J, Zilic M, Martínez L. Patrón pronóstico de invasión serosa (S2) en carcinoma gástrico. Rev Chil Cir. 2002;54:644-8.

48. Norero E, Bustos M, Herrera ME, Cerda J, González P, Ceroni M, et al. Postoperative adjuvant treatment for gastric cancer improves long-term survival after curative resection and D2 lymphadenectomy. Results from a Latin American Center. EJSO.2016;42: 94-102.

49. Manterola C. Cáncer Gástrico Avanzado. Resultados Observados en un Centro Regional Privado de Salud. Int J Med Surg. 2016;3:741-6.

50. Venturelli F, Cárcamo C, Venturelli A, Cárcamo M, Born M, Jara C, et al. Análisis de sobrevida del cáncer gástrico en anillo de sello según profundidad y compromiso linfonodal. Rev Chil Cir. 2008;60:398-402.

51. Csendes A. Medina E, Smok G, Kubo T. Sobrevida de pacientes con cáncer gástrico. II Sobrevida según profundidad aparente de la infiltración tumoral. Rev Med Chile.1975;103:110-2.

52. Flores P, Yáñez E, Cartes R, Grájeda J, Villaseca M, Fernández E, et al. Quimiorradioterapia posoperatoria en el tratamiento del cáncer gástrico. Rev Chil Cir. 2000;52:621-8.

53. Tapia O, Gutiérrez V, Roa JC, Manterola C, Villaseca M, Araya JC. Carcinoma de células en anillo de sello gástrico: Descripción clínico-morfológica y valor pronóstico. Rev Chil Cir. 2010;62:458-64.

54. Csendes A, Cortés S, Guajardo M, Figueroa M. Sobrevida de pacientes con cáncer gástrico etapas IIIC y IV sometidos a cirugía. Parte II de estudio prospectivo 2004-2012. Rev Chil Cir. 2014;66:451-9.

55. Csendes A, Medina E, Smok G, Korn O. Sobrevida a largo plazo de pacientes con cáncer gástrico sometidos a gastrectomía subtotal. Rev Med Chile. 1993;121:138894. 